Luiz Fernando Costa Nascimento ${ }^{1}$

Milena CRISTINA da Silva Almeida ${ }^{2}$

Camila de Moraes Santos Gomes ${ }^{2}$

Artigo Original

Palavras-chave

Mortalidade infantil

Causas de morte

Sistemas de informação geográfica

Análise espacial

Saúde materno-infantil

Keywords

Infant mortality

Cause of death

Geographic information systems Spatial analysis

Maternal and child health

\section{Causas evitáveis e mortalidade neonatal nas microrregiões do estado de São Paulo}

\author{
Neonatal mortality and avoidable causes in the micro \\ regions of São Paulo state
}

\section{Resumo}

OBJETIVO: Identificar padrões espaciais da distribuição da mortalidade neonatal nas microrregiões do estado de São Paulo e verificar o papel das causas evitáveis na composição desse indicador de saúde. MÉTODOS: Este estudo ecológico e exploratório utilizou dados sobre mortalidade neonatal obtidos do Departamento de Informações e Informática do Sistema Único de Saúde (DATASUS) no período entre os anos de 2007 e 2011 . A malha digital das microrregiões do estado de São Paulo foi obtida do Instituto Brasileiro de Geografia e Estatística (IBGE). Foram calculados os coeficientes de Moran, que indicam o grau de correlação espacial, para taxa de mortalidade neonatal total e para taxa por causas evitáveis; foram construídos mapas temáticos com essas taxas e com a diferença entre elas; foi construído o Box Map. RESULTADOS: A taxa de mortalidade neonatal total foi 8,42/1.000 nascidos vivos e a taxa de mortalidade neonatal por causas evitáveis de 6,19/1.000 nascidos vivos. Os coeficientes de Moran (I) para essas taxas foram significativos (valor $\mathrm{p}<0,05$ ) - para a taxa de mortalidade neonatal total $1=0,11$ e para taxa de mortalidade por causas evitáveis $1=0,19-$, e os óbitos neonatais se concentraram na região sudoeste e no Vale do Paraíba. Se as causas evitáveis fossem abolidas, haveria uma redução significativa da taxa média de mortalidade neonatal total, de 8,42 para 2,23 óbitos/1.000 nascidos vivos, representando uma queda de $73 \%$. CONCLUSÃO: Foi possível mostrar que, se as causas de mortes evitáveis fossem de fato abolidas, a taxa de mortalidade neonatal se aproximaria da taxa de países desenvolvidos.

\section{Abstract}

PURPOSE: To identify spatial patterns of neonatal mortality distribution in the micro regions of São Paulo State and verify the role of avoidable causes in the composition of this health indicator. METHODS: This ecological exploratory study used neonatal mortality information obtained from Information System and Information Technology Department of the Brazilian National Healthcare System (DATASUS) in the period between the years 2007 and 2011 . The digital set of micro regions of São Paulo State was obtained from Instituto Brasileiro de Geografia e Estatística (IBGE). Moran Indexes were calculated for the neonatal mortality total rate and rate from avoidable causes; thematic maps were constructed with these rates, as well as the difference between them; and the Box Map was built. RESULTS: The overall neonatal mortality rate was 8.42/1,000 live births and neonatal mortality rate from avoidable causes of 6.19/1,000 live births. Moran coefficients (I) for these rates were significant ( $p$-value $<0.05)$ - for the total rate of neonatal mortality $l=0.11$ and for mortality from preventable causes $1=0.19$-, and neonatal deaths were concentrated in southwest region and the Vale do Paraíba. If preventable causes were abolished, there would be a significant reduction in the average rate of overall neonatal mortality, from 8.42 to 2.23 deaths/ 1,000 live births, representing a decline of 73\%. CONCLUSION: This study demonstrated that neonatal mortality rate would be close to the rates of developed countries if avoidable causes were abolished.
Correspondêncio

Luiz Fernando Costa Nascimento Av. Tiradentes, 500 - Bom Conselho CEP: 12030-180 Taubaté (SP), Brasil

Recebido $28 / 04 / 2014$

Aceito com modificaçōes $23 / 06 / 2014$
Departamento de Medicina da Universidade de Taubaté - Taubaté (SP), Brasil.

'Departamento de Medicina, Universidade de Taubaté - Taubaté (SP), Brasil.

${ }^{2}$ Curso de Medicina, Universidade de Taubaté - Taubaté (SP), Brasil.

Conflito de interesses: não há 


\section{Introdução}

A taxa de mortalidade neonatal (óbitos entre 0 e 27 dias de vida) é um importante indicador de saúde de uma população, refletindo a assistência de saúde prestada à gestante nos períodos pré-natal e parto, como também ao recém-nascido $(\mathrm{RN})$ imediatamente após o nascimento na sala de parto e nas unidades neonatais. É composta por taxa de mortalidade neonatal precoce e tardia, e corresponde a aproximadamente $2 / 3$ dos óbitos infantis ${ }^{1,2}$. Sua taxa no Brasil, em 2011, foi de 9,45 óbitos/1.000 nascidos vivos (NV) e, no estado de São Paulo, 7,91/1.000 NV 3 .

A mortalidade neonatal pode ser determinada por diversos fatores ${ }^{1,2,4}$, mas muitas causas são consideradas evitáveis, ou seja, com o conhecimento e a tecnologia existentes, é possível intervir de maneira eficaz, sem que o paciente chegue a óbito 5 . Mortes por causas evitáveis podem ser importante instrumento de monitorização e avaliação dos serviços de saúde ${ }^{6}$. As mortes evitáveis relacionadas ao recém-nascido são as reduzíveis pela adequada atenção à mulher na gestação, ao parto e ao recém-nascido ${ }^{7}$. Recentemente, Malta et al. ${ }^{8}$ apontaram um decréscimo da ordem de $40 \%$ nas taxas de mortalidade infantil segundo causas evitáveis, mas essa redução se deveu principalmente a ações de imunização; a adequada atenção à mulher no parto e ao recém-nascido também contribuiu para essa redução, ao contrário da atenção à mulher no pré-natal, que aumentou nesse período de dez anos de estudo ${ }^{8}$.

Exemplos de causas de mortalidade neonatal reduzíveis por adequada atenção à mulher na gestação e no parto e ao recém-nascido são descritos em estudos recentes ${ }^{7,8}$.

A localização espacial dos eventos em saúde e os Sistemas de Informações Geográficas (SIG) têm papel destacado e vêm se tornando mais frequentes na literatura da área de saúde pública ${ }^{9-12}$. Analisar a distribuição espacial da mortalidade neonatal pode direcionar ações para melhorias do serviço de saúde a fim de diminuir essa taxa. Estudo semelhante foi realizado no Vale do Paraíba, São Paulo e permitiu identificar aglomerados de mortalidade neonatal na região ${ }^{13}$.

Assim, o objetivo deste estudo foi identificar padrões espaciais da distribuição da mortalidade neonatal no estado de São Paulo e verificar o papel das causas evitáveis na conjuntura desse indicador de saúde.

\section{Métodos}

Trata-se de um estudo ecológico e exploratório com dados sobre mortalidade neonatal e nascidos vivos obtidos do DATASUS ${ }^{3}$ relativos ao período compreendido entre os anos 2007 e 2011 para o estado de São Paulo segundo microrregiões. O estado de São Paulo conta com 63 microrregiões e população de mais de 40 milhões de habitantes. Uma microrregião é um conjunto de municípios com características socioeconômicas semelhantes entre si.

Calculou-se a taxa de mortalidade neonatal por 1.000 nascidos vivos. Os dados sobre óbitos neonatais precoces e tardios, bem como óbitos por causas evitáveis, também foram obtidos do DATASUS ${ }^{14} \mathrm{e}$ analisados com a taxa de mortalidade neonatal total. Foi utilizado o programa TabWin disponibilizado pelo próprio DATASUS, no qual foram feitas as tabelas com as taxas e os respectivos mapas coropléticos. O programa TerraView 4.2.1, de acesso público e desenvolvido pelo INPE (http://www.dpi.inpe.br/), também foi utilizado para calcular o valor do coeficiente de Moran e obter os valores do diagrama de Moran.

A análise espacial identificou autocorrelação espacial dos eventos utilizando o coeficiente Global de Moran (I). Esse índice é utilizado para identificar aglomerados de áreas com riscos semelhantes para ocorrência do desfecho de interesse, pode variar de $-1 \mathrm{a}+1$. Valores próximos de zero indicam a inexistência de autocorrelação espacial significativa entre os valores dos objetos e seus vizinhos. Valores positivos indicam que as microrregiões vizinhas são similares entre si, e valores negativos que não são ${ }^{15}$.

Foi realizada a construção dos mapas da taxa de mortalidade total e por causas evitáveis, e depois foi construído o mapa da diferença entre a taxa de mortalidade neonatal e por causas evitáveis (situação ideal), além do Box Map. O Box Map é construído com informações do diagrama de Moran, o qual é uma maneira adicional de visualizar a dependência espacial, pois permite analisar o comportamento da variabilidade espacial. A ideia é comparar os valores do atributo em uma área com a média dos seus vizinhos, construindo um gráfico bidimensional que é dividido em quatro quadrantes os quadrantes 1 (Q1) e $2(\mathrm{Q} 2)$ costumam ser objetos de discussão e podem ser interpretados como Q1 (valores positivos, médias positivas) e Q2 (valores negativos, médias negativas): indicam pontos de associação espacial positiva, no sentido que uma localização tem vizinhos com valores semelhantes ${ }^{15}$.

O nível de significância adotado foi $\alpha=5 \%$. Este trabalho foi aprovado pelo Comitê de Ética em Pesquisa da Universidade de Taubaté sob o número 045/11.

\section{Resultados}

No período, foram registrados 3.007.250 nascidos vivos, com 17.999 óbitos neonatais precoces $(5,98 / 1.000 \mathrm{NV}$, variando de 3,47 a 70,80$)$ e 7.332 óbitos neonatais tardios $(2,44 / 1.000 \mathrm{NV}$, variando de 0,85 a 13,27). Houve 18.621 óbitos por causas evitáveis com taxa de 6,19/1.000 NV, variando de 3,76 a 12,75. Todos os coeficientes de Moran Global (I) mostraram 
significância estatística - para a taxa de mortalidade neonatal total $\mathrm{I}=0,11$ e para taxa de mortalidade por causas evitáveis $\mathrm{I}=0,19$.

O mapa temático com as taxas de mortalidade neonatal total (Figura 1) mostrou valores menores na região norte e central do estado, e maiores nas regiões do Vale do Paraíba e sudoeste do estado, destacando-se as microrregiões de Capão Bonito, Itapeva, Itanhaém, Santos, Campos do Jordão e Guaratinguetá, e Araçatuba, Adamantina e Votuporanga na região noroeste. As microrregiões com as menores taxas são Barretos, São José do Rio Preto, Catanduva, Jaboticabal e Ribeirão Preto no norte do estado, e São Carlos, Mogi-Mirim, Botucatu e Campinas nas regiões central e sudeste.

O mapa da taxa de mortalidade neonatal por causas evitáveis (reduzível por atenção à gestante, ao parto e ao recém-nascido) (Figura 2) mostrou o mesmo padrão de distribuição da Figura 1, com as maiores taxas concentrando-se no Vale do Paraíba, litoral e sudoeste do estado, destacando-se as microrregiões de Campos do Jordão, Paraibuna/Paraitinga, Guaratinguetá, Santos, Itapeva e Capão Bonito.

A diferença entre a taxa de mortalidade neonatal total e a taxa de mortalidade neonatal por causas evitáveis reduzível por adequada atenção à gestante mostrou uma queda de $34 \%$ na taxa, que foi de 8,42 para 5,53 óbitos/1.000 NV, considerando os valores médios.

A diferença entre a taxa de mortalidade neonatal total e a taxa de mortalidade neonatal por causas evitáveis reduzível pela adequada atenção no parto foi de 8,42 para 7,42 óbitos/1.000 NV, uma redução de 12\%, considerando os valores médios.

Quanto à adequada atenção ao recém-nascido, a diferença entre a taxa de mortalidade neonatal total e a taxa de mortalidade neonatal por causas evitáveis reduzível pela adequada atenção ao recém-nascido foi de 8,42 para 6,12 , queda de $27 \%$ considerando os valores médios (mapas não mostrados).

O mapa da diferença entre a taxa de mortalidade neonatal total e a taxa de mortalidade por causas evitáveis mostra as taxas de mortalidade neonatal se as causas evitáveis fossem abolidas (Figura 3). Houve uma redução significativa da taxa média, de 8,42 para 2,23 óbitos/1.000 $\mathrm{NV}$, representando uma queda de $73 \%$.

O Box Map (Figura 4) mostra as regiões com alta prioridade de atenção, que são as de cor mais escura. Essas microrregiões estão localizadas no sul, litoral e Vale do Paraíba, destacando-se as microrregiões de Itapeva, Capão Bonito, Registro, Santos, Itanhaém, Caraguatatuba, Paraibuna/Paraitinga, Campos do Jordão e Guaratinguetá.

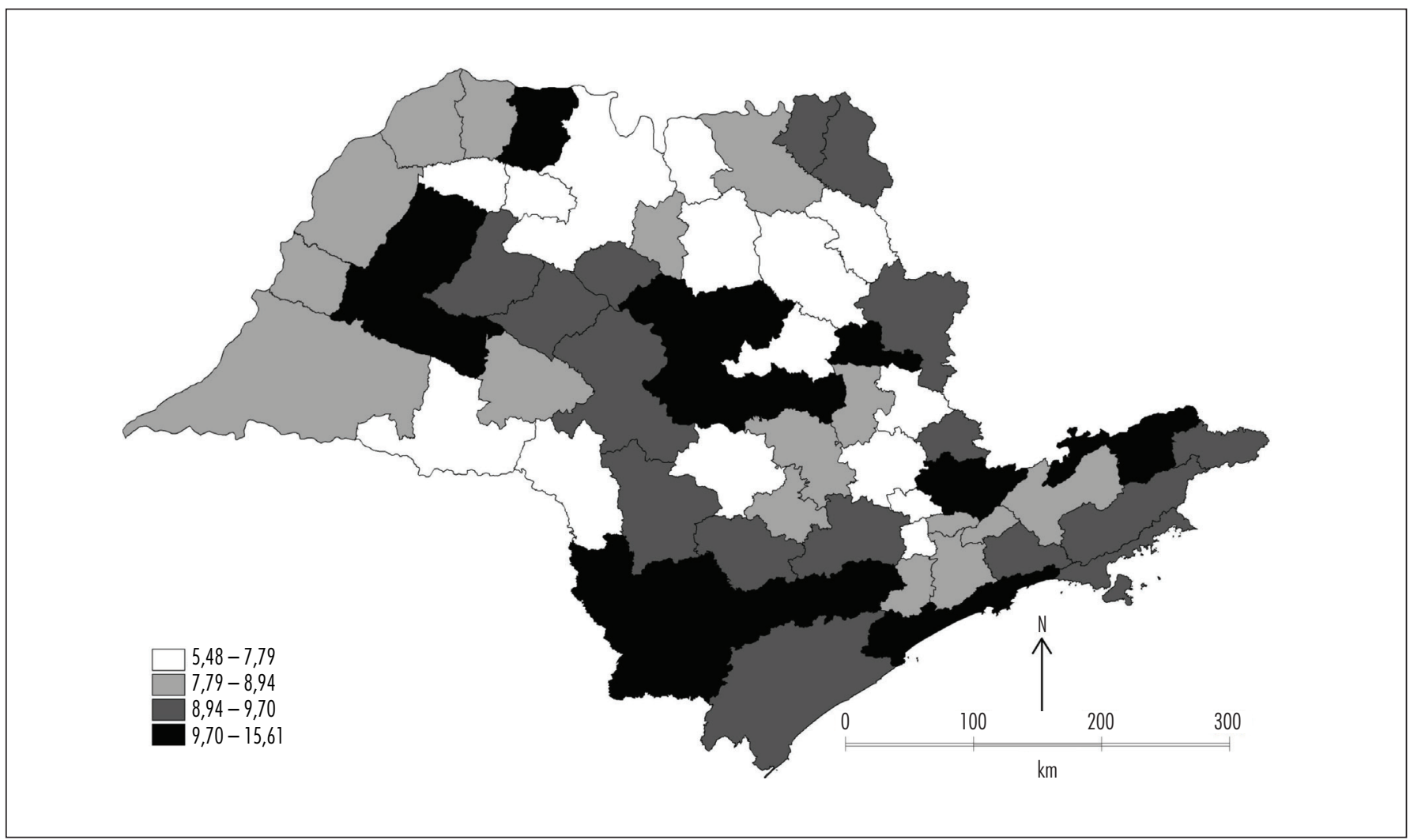

Figura 1. Mapa temático com as taxas de mortalidade neonatal total representadas por cores e divididas em quatro faixas: 5,79 a 7,$79 ; 7,79$ a 8,$94 ; 8,94$ a 9,70 e 9,70 a 15,61 por 1.000 nascidos vivos, segundo microrregiões, São Paulo, 2007-2011 


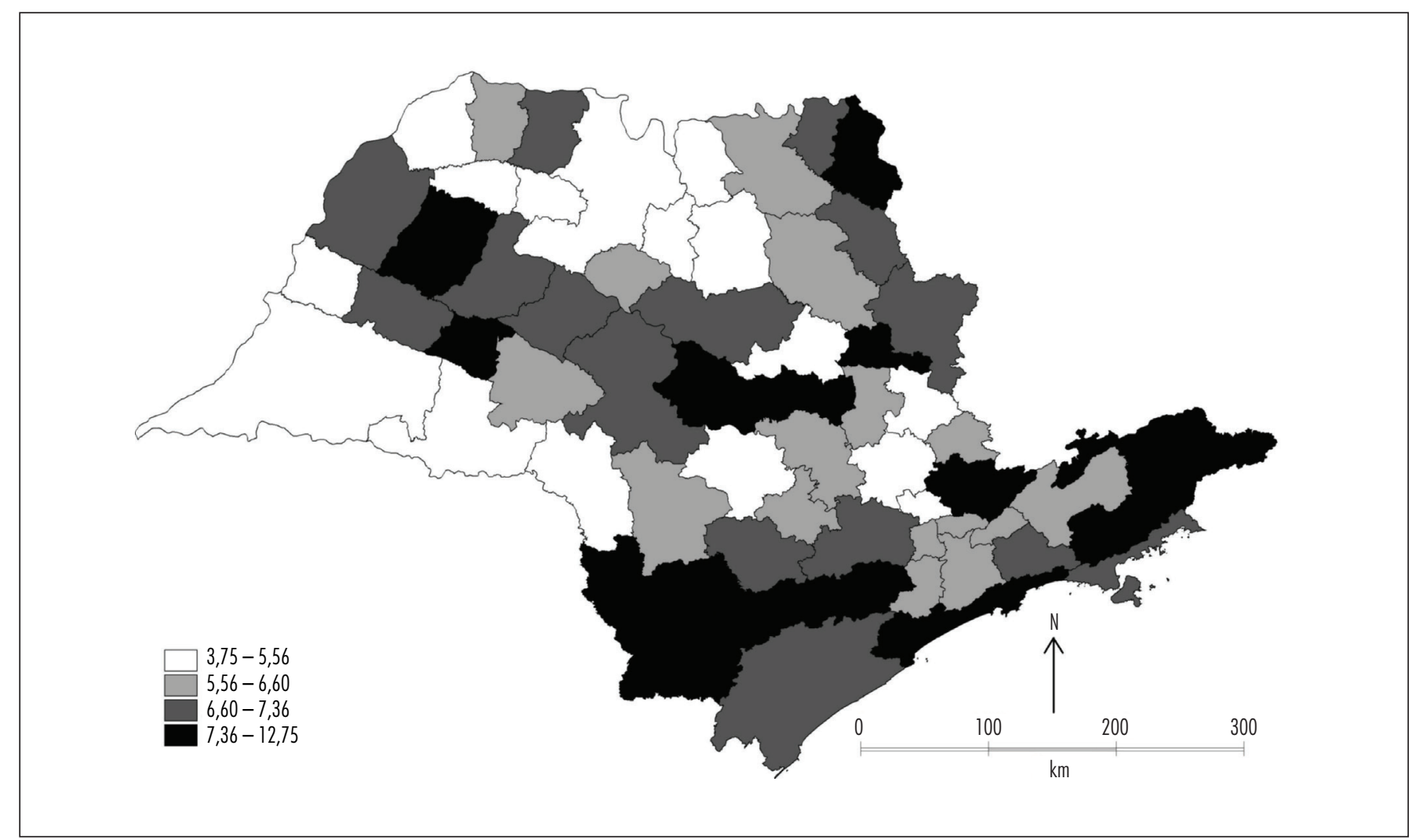

Figura 2. Mapa temático com as taxas de mortalidade por causas evitáveis representadas por cores e divididas em quatro faixas: 3,75 a 5,56; 5,56 a 6,60; 6,60 a 7,36 e 7,36 a 12,75 por 1.000 nascidos vivos, segundo microrregiões, São Paulo, 2007-201 1

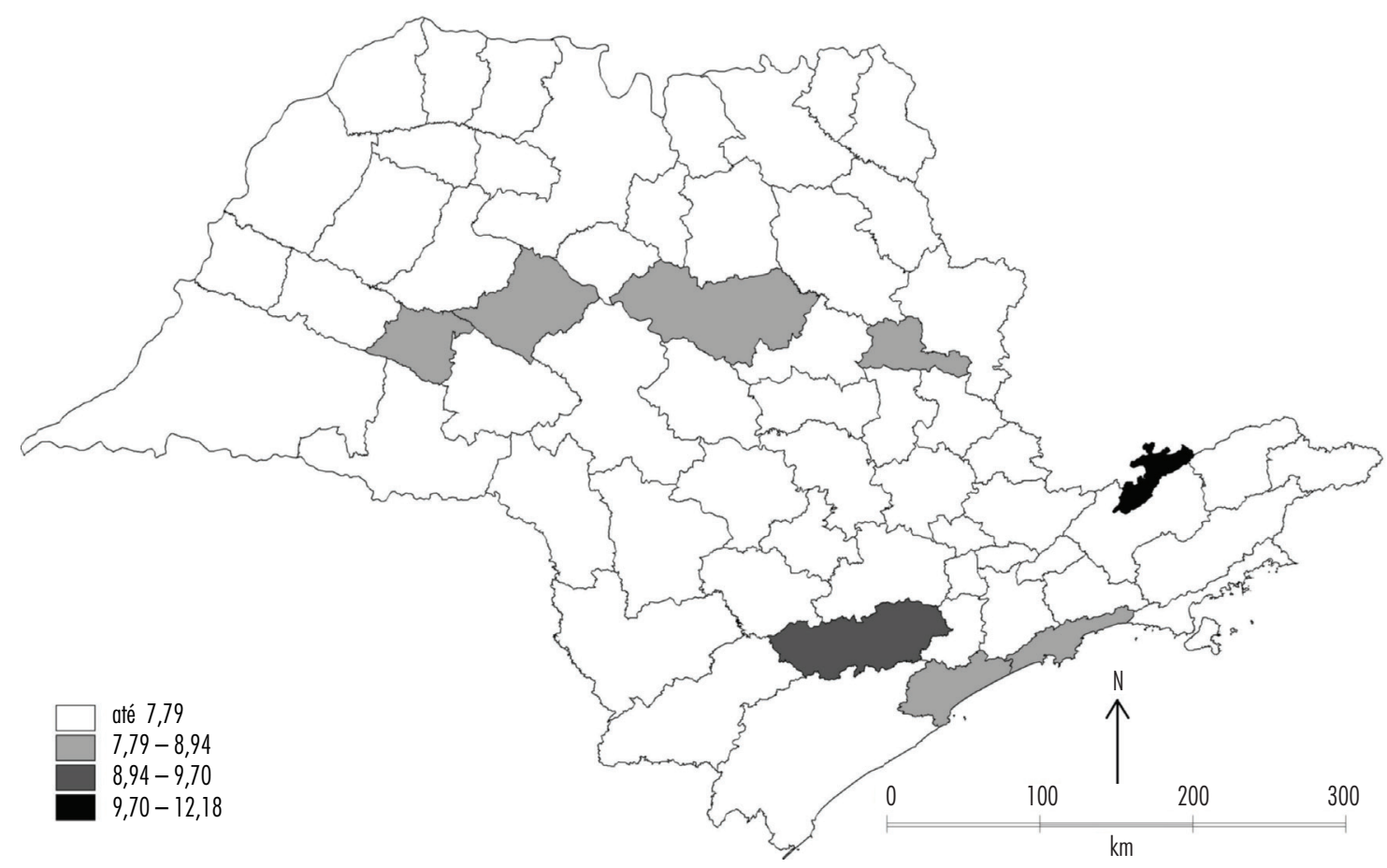

Figura 3. Mapa temático com a diferença entre as taxas de mortalidade neonatal total e por causas evitáveis representadas por cores e divididas em quatro faixas: até 7,$79 ; 7,79$ a 8,94; 8,94 a 9,70 e 9,70 a 12,18 por 1.000 nascidos vivos, segundo microrregiões, São Paulo, $2007-2011$ 


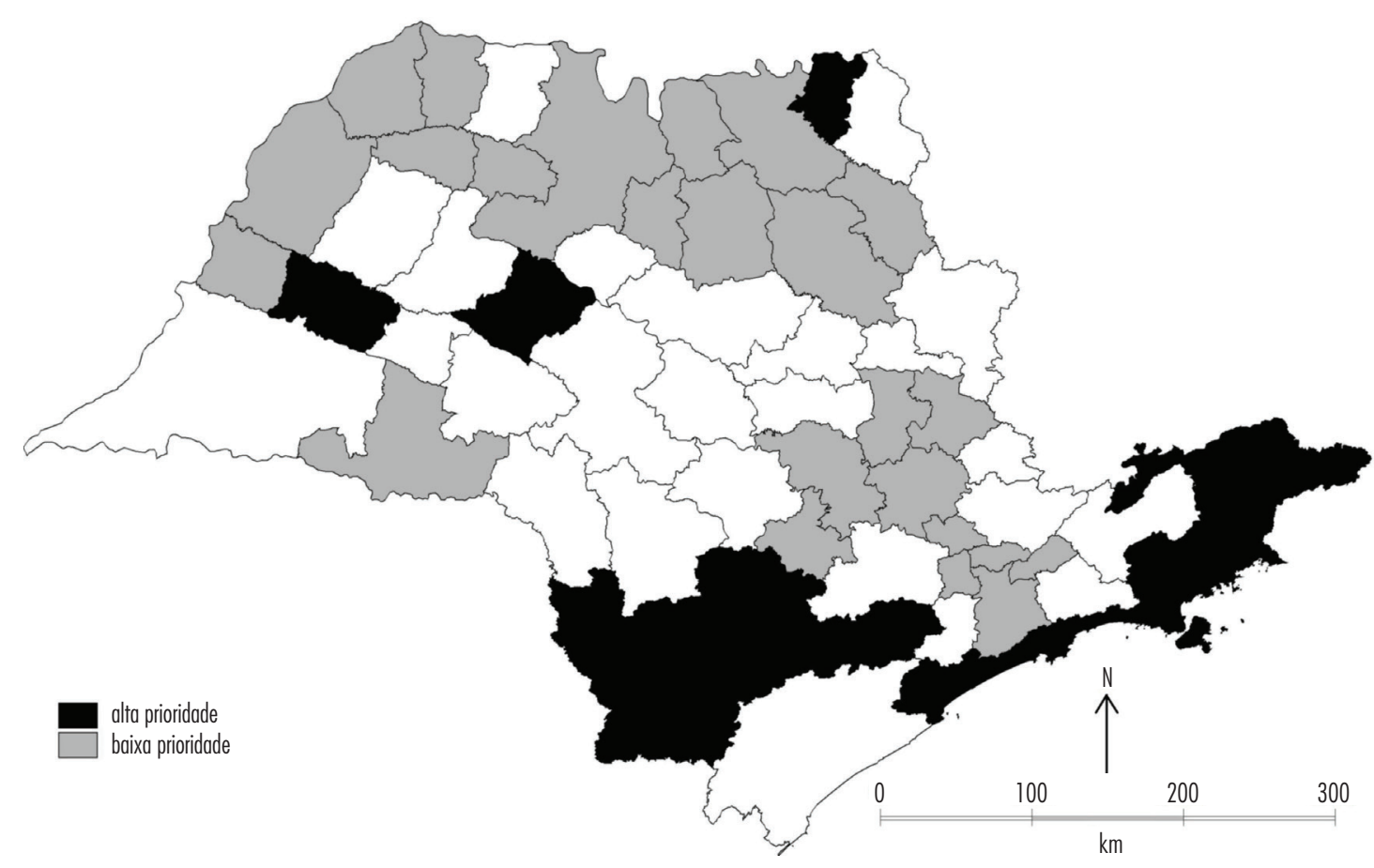

Figura 4. Box Map mostrando microrregiões com alta e baixa prioridade de intervenção, segundo microrregiões, São Paulo, 2007-201 1

\section{Discussão}

Este é o primeiro estudo realizado com o objetivo de identificar espacialmente a importância das causas evitáveis na mortalidade neonatal, sendo possível identificar microrregiões onde medidas devem ser tomadas para melhorar esse índice.

O sul do estado é considerado uma região mais pobre, portanto é bem possível que as condições de saúde não sejam adequadas, o que pode contribuir para taxas mais altas de mortalidade neonatal nas microrregiões de Itapeva, Capão Bonito, Piedade e Itanhaém e moderadas taxas em Registro.

O Vale do Paraíba, como já mostraram Nascimento et al. ${ }^{13}$, apresentou elevadas taxas de mortalidade neonatal. Uma provável justificativa seria o atendimento insatisfatório dispensado à gestante e ao recém-nascido, já que se trata de uma região cortada por uma rodovia importante, a rodovia Dutra, e conta com serviços hospitalares e ambulatoriais. Porém, é importante salientar que esses serviços se concentram em poucos municípios (São José dos Campos, Taubaté e Guaratinguetá) e, muitas vezes, o acesso a eles se torna prejudicado por conta da distância que o indivíduo tem que percorrer.

No sudeste do estado, onde também foram encontradas microrregiões com elevadas taxas, os serviços de saúde acessíveis e de boa qualidade deveriam ser capazes de reconhecer as mortes evitáveis e programar medidas para reduzi-las. Isso indica que fatores socioeconômicos podem não ser os únicos responsáveis pela alta taxa de mortalidade neonatal, já que microrregiões supostamente mais ricas também as apresentam.

A região norte, por sua vez, apresentou baixas taxas, possivelmente por causa da existência de grandes centros de referência em saúde, como em Ribeirão Preto, Barretos e São José do Rio Preto, mostrando que há melhor atenção às gestantes e aos recém-nascidos.

Quando foi considerada a taxa total de mortalidade neonatal menos a taxa de mortalidade neonatal por causas evitáveis, observou-se mais de $70 \%$ de redução nos óbitos, o que mostra que a dificuldade ao acesso, a desorganização e a fragmentação do sistema de saúde, bem como inadequações técnico-científicas da assistência, são dificuldades encontradas no país quanto à atenção à gestante e ao RN.

A mortalidade por causas evitáveis reduzíveis por adequado atendimento à gestante é o principal componente da mortalidade neonatal por causas evitáveis, seguido pelas reduzíveis pela adequada atenção ao RN e depois pelo atendimento no parto. Em relação ao pré-natal, a hierarquização, a garantia do acesso e a qualidade do atendimento, e não apenas a quantidade de consultas, são pontos-chave na melhoria da atenção ${ }^{4,16}$. 
A qualidade da assistência pré-natal e perinatal está diretamente relacionada ao óbito neonatal, e mesmo neonatos prematuros de muito baixo peso são passíveis de intervenção ${ }^{17}$.

Estudo retrospectivo realizado em municípios do sul-mato-grossense sobre mortalidade neonatal mostrou que as mortes evitáveis e reduzíveis perfizeram $73,1 \%$, na maior parte $(69,5 \%)$ em decorrência de inadequada atenção à mulher na gestação e no parto e ao recém-nascido, o que também vai de acordo com os resultados deste estudo ${ }^{18}$.

Em um hospital de referência em Pernambuco, foram avaliados os óbitos infantis e concluiu-se que a maioria dos óbitos estava classificada entre as causas reduzíveis por medidas de atenção à saúde, com mortalidade proporcional de 77,4\% (infantil), 75,3\% (neonatal) e 60,1\% (pós-neonatal); quase metade eram neonatos, dos quais $31 \%$ eram reduzíveis por diagnóstico e tratamento precoces, o que sugere problemas de acesso aos serviços de saúde, cobertura e/ou na qualidade da assistência prestada ${ }^{19}$.

Estudo ecológico para testar as associações entre a mortalidade infantil por causas evitáveis no Brasil com indicadores socioeconômicos e de serviços e investimentos em saúde verificou que os municípios que compuseram os quartis com maior mortalidade infantil por causas evitáveis apresentaram também menor Índice de Desenvolvimento Humano Municipal, mostrando o papel protagonista das condições socioeconômicas e de investimentos em saúde sobre a mortalidade infantil por causas evitáveis ${ }^{4}$.

A análise espacial é uma grande ferramenta deste estudo, apontando as regiões que necessitam de uma atenção maior. No Rio de Janeiro, a análise espacial foi utilizada para identificar fatores explicativos das variações espaciais da taxa de mortalidade neonatal precoce; as variáveis que melhor explicaram os aglomerados espaciais foram: "proporção de mães adolescentes", "proporção de pessoas residentes em favelas em 1996" e "proporção de chefes com rendimento até um salário mínimo" 20 .

Não foram analisadas as distribuições de baixo peso e prematuridade, fatores que contribuem para a mortalidade neonatal. O nível de desenvolvimento de cada microrregião não foi relacionado, mesmo tendo em vista a importância deste na mortalidade neonatal, porque não existem esses dados por microrregião, apenas por municípios. Além desses pontos assinalados, não foi possível identificar possíveis fatores de risco, pois, no estudo ecológico, a unidade de observação é a população e não o indivíduo, e os dados incluídos na análise não discriminam individualmente cada informação do recém-nascido quanto a peso, duração da gestação, idade materna, entre outras variáveis. Esses fatores assinalados podem ser considerados limitações do estudo, além das subnotificações dos óbitos.

No entanto, a força do estudo está na identificação de microrregiões com altas taxas de mortalidade neonatal e altas taxas de mortalidade neonatal por causas evitáveis, assim como aquelas com alta prioridade de atenção.

Do exposto acima, se as mortes evitáveis redutíveis por atenção à gestante no pré-natal e no parto e ao recém-nascido fossem de fato evitadas, a taxa de mortalidade neonatal se aproximaria da taxa de países desenvolvidos. Para melhorias nesse índice, uma atenção integral e de qualidade à saúde infantil desde o período pré-natal se faz necessária. A identificação de microrregiões com alta prioridade de intervenção poderá permitir aos gestores regionais programar políticas de saúde à gestante e ao recém-nascido.

\section{Referências}

1. Nascimento RM, Leite AJ, Almeida NM, Almeida PC, Silva CF. Determinantes da mortalidade neonatal: estudo caso-controle em Fortaleza, Ceará, Brasil. Cad Saúde Pública. 2012;28(3):559-72.

2. Paulucci RS, Nascimento LF. Mortalidade neonatal em Taubaté um estudo caso-controle. Rev Paul Pediatr. 2007;25(4):358-63.

3. Brasil. Ministério da Saúde. DATASUS [Internet]. Informações de Saúde. Estatísticas vitais. Mortalidade e nascidos vivos: nascidos vivos desde 1994. Brasília (DF): Ministério da Saúde; 2014 [citado 2014 Abr 16]. Disponível em: <http://www2.datasus.gov.br/DATASUS/index.php?area=02>

4. Ribeiro AM, Guimarães M, Lima MC, Sarinho SW, Coutinho SB. Fatores de risco para mortalidade neonatal em crianças com baixo peso ao nascer. Rev Saúde Pública. 2009;43(2):246-55.

5. Boing $A F$, Boing AC. Mortalidade infantil por causas evitáveis no Brasil: um estudo ecológico no período 2000-2002. Cad Saúde Pública. 2008;24(2):447-55.
6. Malta DC, Duarte EC, Escalante JJ, Almeida MF, Sardinha LM, Macário EM, et al. Mortes evitáveis em menores de um ano, Brasil, 1997 a 2006: contribuições para a avaliação de desempenho do Sistema Único de Saúde. Cad Saúde Pública. 2010;26(3):481-91.

7. Malta DC, Duarte EC, Almeida MF, Dias MA, Morais Neto OL, Moura L, et al. Lista de causas de mortes evitáveis por intervenções do Sistema Único de Saúde do Brasil. Epidemiol Serv Saúde. 2007; 16(4):233-44.

8. Malta DC, Sardinha LM, Moura L, Lansky S, Leal MC, Szwarcwald $\mathrm{CL}$, et al. Atualização da lista de causas de mortes evitáveis por intervenções do Sistema Único de Saúde do Brasil. Epidemiol Serv Saúde. 2010;19(2):173-6.

9. Ratmanov P, Mediannikov O, Raoult D. Vectorborne diseases in West Africa: geographic distribution and geospatial characteristics. Trans R Soc Trop Med Hyg. 2013; 107(5):273-84. 
10. Carvalho MS, Santos RS. Análise de dados espaciais em saúde pública: métodos, problemas, perspectivas. Cad Saúde Pública. 2005;21 (2):361-78.

11. Antunes FP, Costa MC, Paim JS, Vieira-da-Silva LM, Cruz AA, Natividade $M$, et al. Desigualdades sociais na distribuição espacial das hospitalizações por doenças respiratórias. Cad Saúde Pública. 2013;29(7):1346-56.

12. Clements AC, Reid HL, Kelly GC, Hay SI. Further shrinking the malaria map: how can geospatial science help to achieve malaria elimination? Lancet Infect Dis. 2013;13(8):709-18.

13. Nascimento LF, Batista GT, Dias NW, Catelani CS, Becker D, Rodrigues L. Análise espacial da mortalidade neonatal no Vale do Paraíba, 1999 a 2001. Rev Saúde Pública. 2007;41(1):94-100.

14. Brasil. Ministério da Saúde. DATASUS [Internet]. Informações de Saúde. Estatísticas vitais. Mortalidade e nascidos vivos: óbitos infantis desde 1996. Brasília (DF): Ministério da Saúde; 2014 [citado $2014 \mathrm{abr}$ 16]. Disponível em: <http://tabnet.datasus. gov.br/cgi/tabcgi.exe? sim/cnv/inf10sp.def>

15. Câmara G, Carvalho MS, Cruz OG, Correa V. Análise de dados de área. In: Druck S, Carvalho MS, Câmara G, Monteiro AV, editores.
Análise espacial de dados geográficos. Brasília (DF): Embrapa; 2004 [citado 2014 abr 02]. Disponível em: <http://www.dpi. inpe.br/gilberto/livro/analise/cap5-areas.pdf>

16. Almeida SD, Barros MB. Atenção à saúde e mortalidade neonatal: estudo caso-controle realizado em Campinas, São Paulo. Rev Bras Epidemiol. 2004;7(1):22-35

17. Almeida MF, Guinsburg R, Martinez FE, Procianoy RS, Leone CR, Marba STM, et al. Fatores perinatais associados ao óbito precoce em prematuros nascidos nos centros da Rede Brasileira de Pesquisas Neonatais. J Pediatr (Rio J). 2008;84(4):300-7.

18. Gastaud AL, Honer MR, Cunha RV. Mortalidade infantil e evitabilidade em Mato Grosso do Sul, Brasil, 2000 a 2002. Cad Saúde Pública. 2008;24(7):1631-40.

19. Vidal SA, Frias PG, Barreto FM, Vanderlei LC, Felisberto E. Óbitos infantis evitáveis em hospital de referência estadual do Nordeste brasileiro. Rev Bras Saúde Matern Infant. 2003;3(3):281-9.

20. Andrade CL, Szwarcwald CL. Análise espacial da mortalidade neonatal precoce no Município do Rio de Janeiro, 1995-1996. Cad Saúde Pública. 2001;17(5):1199-210. 